\title{
Additive and Multiplicative Perturbations of Exponentially Dichotomous Operators on General Banach Spaces
}

\author{
Cornelis V.M van der Mee and André C.M. Ran
}

To Israel Gohberg on the occasion of his $75^{\text {th }}$ birthday

\begin{abstract}
Recent perturbation results for exponentially dichotomous operators are generalized, in part by replacing compactness conditions on the perturbation by resolvent compactness. Both additive and multiplicative perturbations are considered.

Mathematics Subject Classification (2000). Primary 47D06; Secondary 47A55.

Keywords. block operator, exponential dichotomy, semigroup perturbation, Riccati equation.
\end{abstract}

\section{Introduction}

In [14] perturbation results for exponentially dichotomous operators on Banach spaces were discussed. In this paper we continue the investigation started in that paper.

Recall that an exponentially dichotomous operator is a direct sum $A_{0} \dot{+}\left(-A_{1}\right)$, in which $A_{0}$ and $A_{1}$ are generators of exponentially decaying $C_{0}$-semigroups. Such operators were introduced in $[2,3]$ in connection with convolution equations on the half-line. Operators of this type also occur in various other applications, see, e.g., $[7,8,9,10,11]$.

Perturbation results for exponentially dichotomous operators were already studied in [2], where additive perturbations were considered. Results in this direction were later obtained for more particular operators on Hilbert spaces in $[8,9,10,11]$. In [14] the authors considered additive perturbations for exponentially dichotomous operators on Banach spaces. Multiplicative perturbations were studied in $[7,13]$.

The research leading to this article was supported in part by MIUR under the COFIN grants 2002014121 and 2004015437, and by INdAM. 
In this article we accomplish the following two tasks. First we generalize the main results on additive and bounded perturbations of exponentially dichotomous operators derived in [14] by requiring the corresponding bisemigroup multiplied from the right by a bounded additive perturbation to be continuous in the operator norm, except possibly as $t \rightarrow 0^{ \pm}$. This greatly simplifies the treatment in [14], where it is assumed that either the corresponding bisemigroup itself is continuous in the operator norm (except possibly as $t \rightarrow 0^{ \pm}$) or the perturbation is a compact operator. We shall prove a lemma that will allow us to use the same proofs as in [14] and to refer to [14] for these proofs. Secondly, for exponentially dichotomous operators having bounded analytic constituent semigroups, we study perturbations obtained by multiplying the given exponentially dichotomous operator from the right by a compact perturbation of the identity. We shall prove that the newly obtained operator is exponentially dichotomous and has bounded analytic constituent semigroups. We thus generalize results obtained before in [7] in a Hilbert space setting. All of our results will be derived in general complex Banach spaces, including those on Riccati equations.

The main body of this paper consists of two sections. In Section 2 we indicate how one of the main results of [14] can be generalized to the present setting without changing its proof and discuss the consequences of this result for canonical factorization and for block operators. In Section 3 we study perturbations of analytic bisemigroup generators. We refer to the introduction of [14] for a more comprehensive discussion of the existing literature.

Let us introduce some notations. We let $\mathbb{R}^{ \pm}$stand for the right (left, resp.) half-line, including the point at zero. For two complex Banach spaces $\mathcal{X}$ and $\mathcal{Y}$, we let $\mathcal{L}(\mathcal{X}, \mathcal{Y})$ stand for the Banach spaces of all bounded linear operators from $\mathcal{X}$ into $\mathcal{Y}$. We write $\mathcal{L}(\mathcal{X})$ instead of $\mathcal{L}(\mathcal{X}, \mathcal{X})$.

Let $\mathcal{X}$ be a complex Banach space and $E$ an interval of the real line $\mathbb{R}$. Then $L^{p}(E ; \mathcal{X})$ denotes the Banach space of all strongly measurable functions $\phi: E \rightarrow \mathcal{X}$ such that $\|\phi(\cdot)\|_{\mathcal{X}} \in L^{p}(E)$, endowed with the $L^{p}$-norm, and $C_{0}(E ; \mathcal{X})$ stands for the Banach space of all bounded continuous functions $\phi: E \rightarrow \mathcal{X}$ which vanish at infinity if $E$ is unbounded, endowed with the supremum norm. In particular, $C_{0}\left(\mathbb{R}^{-} ; \mathcal{X}\right) \dot{+} C_{0}\left(\mathbb{R}^{+} ; \mathcal{X}\right)$ is the Banach space of all bounded continuous functions $\phi: \mathbb{R} \rightarrow \mathcal{X}$ which vanish at $\pm \infty$ and may have a jump discontinuity in zero.

\section{Bisemigroups and their perturbations}

\subsection{Bisemigroup perturbation results}

A $C_{0}$-semigroup $(T(t))_{t \geq 0}$ on a complex Banach space $\mathcal{X}$ is called uniformly exponentially stable if

$$
\|T(t)\| \leq M e^{-\varepsilon t}, \quad t \geq 0
$$

for certain $M, \varepsilon>0$. 
A closed and densely defined linear operator $-S$ on a Banach space $\mathcal{X}$ is called exponentially dichotomous [2] if for some projection $P$ commuting with $S$, the restrictions of $S$ to $\operatorname{Im} P$ and of $-S$ to Ker $P$ are the infinitesimal generators of exponentially decaying $C_{0}$-semigroups. We then define the bisemigroup generated by $-S$ as

$$
E(t ;-S)=\left\{\begin{array}{cc}
e^{-t S}(I-P), & t>0 \\
-e^{-t S} P, & t<0 .
\end{array}\right.
$$

Its separating projection $P$ is given by $P=-E\left(0^{-} ;-S\right)=I_{\mathcal{X}}-E\left(0^{+} ;-S\right)$. One easily verifies the existence of $\varepsilon>0$ such that $\{\lambda \in \mathbb{C}:|\operatorname{Re} \lambda| \leq \varepsilon\}$ is contained in the resolvent set $\rho(S)$ of $S$ and for every $x \in \mathcal{X}$

$$
(\lambda-S)^{-1} x=-\int_{-\infty}^{\infty} e^{\lambda t} E(t ;-S) x d t, \quad|\operatorname{Re} \lambda| \leq \varepsilon .
$$

As a result, for every $x \in \mathcal{X}$ we have $\left\|(\lambda-S)^{-1} x\right\| \rightarrow 0$ as $\lambda \rightarrow \infty$ in $\{\lambda \in \mathbb{C}$ : $\left.|\operatorname{Re} \lambda| \leq \varepsilon^{\prime}\right\}$ for some $\varepsilon^{\prime} \in(0, \varepsilon]$. We call the restrictions of $e^{-t S}$ to Ker $P$ and of $e^{t S}$ to Im $P$ the constituent semigroups of the exponentially dichotomous operator $-S$. Observe that $\left\{x \in \mathcal{X}:(\lambda-S)^{-1} x\right.$ is analytic for $\left.\operatorname{Re} \lambda<0\right\}=\operatorname{Ker} P$, and $\left\{x \in \mathcal{X}:(\lambda-S)^{-1} x\right.$ is analytic for $\left.\operatorname{Re} \lambda>0\right\}=\operatorname{Im} P$.

Before deriving our main perturbation result, we prove the following lemma. Note that Theorem 3 of [14] is an immediate consequence of this lemma.

Lemma 2.1. Let $-S_{0}$ be exponentially dichotomous, $\Gamma$ a bounded operator such that $E\left(t ;-S_{0}\right) \Gamma$ is norm continuous in $0 \neq t \in \mathbb{R}$, and $-S=-S_{0}+\Gamma$, where $\mathcal{D}(S)=$ $\mathcal{D}\left(S_{0}\right)$. Suppose the strip $\{\lambda \in \mathbb{C}:|R e \lambda|<\varepsilon\}$ is contained in the resolvent set of $S$ for some $\varepsilon>0$. Then $-S$ is exponentially dichotomous. Moreover, $E(t ;-S) \Gamma$ is norm continuous in $0 \neq t \in \mathbb{R}$ with norm continuous limits as $t \rightarrow 0^{ \pm}$.

Proof. There exists $\varepsilon>0$ such that

$$
\int_{-\infty}^{\infty} e^{\varepsilon|t|}\left\|E\left(t ;-S_{0}\right)\right\| d t<\infty
$$

Using the resolvent identity

$$
(\lambda-S)^{-1}-\left(\lambda-S_{0}\right)^{-1}=-\left(\lambda-S_{0}\right)^{-1} \Gamma(\lambda-S)^{-1}, \quad|\operatorname{Re} \lambda| \leq \varepsilon,
$$

for some $\varepsilon>0$, we obtain the convolution integral equation

$$
E(t ;-S) x-\int_{-\infty}^{\infty} E\left(t-\tau ;-S_{0}\right) \Gamma E(\tau ;-S) x d \tau=E\left(t ;-S_{0}\right) x
$$

where $x \in \mathcal{H}$ and $0 \neq t \in \mathbb{R}$. By assumption, in (2.5) the convolution kernel $E\left(\cdot ;-S_{0}\right) \Gamma$ is continuous in the norm except for a jump discontinuity in $t=0$. Further, (2.3) implies that $e^{\varepsilon|\cdot|} E\left(\cdot ;-S_{0}\right) \Gamma$ is Bochner integrable.

The symbol of the convolution integral equation (2.5), which equals $I_{\mathcal{H}}+$ $\left(\lambda-S_{0}\right)^{-1} \Gamma=\left(\lambda-S_{0}\right)^{-1}(\lambda-S)$, tends to $I_{\mathcal{H}}$ in the norm as $\lambda \rightarrow \infty$ in the strip $|\operatorname{Re} \lambda| \leq \varepsilon$, because of the Riemann-Lebesgue lemma. Thus there exists $\varepsilon_{0} \in(0, \varepsilon]$ such that the symbol only takes invertible values on the strip $|\operatorname{Re} \lambda| \leq \varepsilon_{0}$. By 
the Bochner-Phillips theorem ([4], also [6]), the convolution equation (2.5) has a unique solution $u(\cdot ; x)=E(\cdot ;-S) x$ with the following properties:

1) $E(\cdot ;-S)$ is strongly continuous, except for a jump discontinuity at $t=0$,

2) $\int_{-\infty}^{\infty} e^{\varepsilon_{0}|t|}\|E(t ;-S)\| d t<\infty$; hence $E(\cdot ;-S)$ is exponentially decaying,

$3)$ the identity (2.2) holds.

As a result [2], $-S$ is exponentially dichotomous.

We now present our main perturbation result. Note that Theorem 2 of [14] is an immediate consequence of this result.

Theorem 2.2. Let $-S_{0}$ be exponentially dichotomous, $\Gamma$ a bounded operator such that the operator $\left(\lambda-S_{0}\right)^{-1} \Gamma$ is compact for imaginary $\lambda$, and $-S=-S_{0}+\Gamma$, where $\mathcal{D}(S)=\mathcal{D}\left(S_{0}\right)$. Suppose the imaginary axis is contained in the resolvent set of $S$. Then $-S$ is exponentially dichotomous. Moreover, $E(t ;-S)-E\left(t ;-S_{0}\right)$ is a compact operator, also in the limits as $t \rightarrow 0^{ \pm}$.

Proof. It suffices to prove that $E\left(t ;-S_{0}\right) \Gamma$ is a compact operator for $0 \neq t \in \mathbb{R}$. This would imply that (1) $E\left(t ;-S_{0}\right) \Gamma$ is norm continuous in $0 \neq t \in \mathbb{R}$ with norm continuous limits as $t \rightarrow 0^{ \pm}$, and (2) the symbol $I_{\mathcal{H}}+\left(\lambda-S_{0}\right)^{-1} \Gamma=$ $\left(\lambda-S_{0}\right)^{-1}(\lambda-S)$ of the convolution integral equation (2.5) tends to $I_{\mathcal{H}}$ in the norm as $\lambda \rightarrow \infty$ in the strip $|\operatorname{Re} \lambda| \leq \varepsilon$. In combination with the absence of imaginary spectrum of $S$, the latter would imply that the strip $\left\{\lambda \in \mathbb{C}:|\operatorname{Re} \lambda|<\varepsilon_{0}\right\}$ is contained in the resolvent set of $S$ for some $\varepsilon_{0}>0$. Theorem 2.2 would then be immediate from Lemma 2.1.

By analytic continuation, we easily prove that $\left(\lambda-S_{0}\right)^{-1} \Gamma$ is a compact operator on a strip $\{\lambda \in \mathbb{C}:|\operatorname{Re} \lambda| \leq \varepsilon\}$ for some $\varepsilon>0$. Thus $\left(\lambda-S_{0}\right)^{-1} E\left(0^{+},-S_{0}\right) \Gamma$ is analytic and compact operator valued for $\operatorname{Re} \lambda<\varepsilon$, while $\left(\lambda-S_{0}\right)^{-1} E\left(0^{-},-S_{0}\right) \Gamma$ is analytic and compact operator valued for $\operatorname{Re} \lambda>-\varepsilon$.

Now it is well known ([5], Corollary III 5.5) that

$$
E\left(t ;-S_{0}\right) x= \begin{cases}\lim _{n \rightarrow \infty}\left(I+\frac{t}{n} S_{0}\right)^{-n} E\left(0^{+} ;-S_{0}\right) x, & t>0, \\ \lim _{n \rightarrow \infty}\left(I+\frac{t}{n} S_{0}\right)^{-n} E\left(0^{-} ;-S_{0}\right) x, & t<0 .\end{cases}
$$

uniformly in $x$ on relatively compact sets. Since for every $0 \neq t \in \mathbb{R}$ we have that $\left(I+\frac{t}{n} S_{0}\right)^{-1} \Gamma$ is compact for sufficiently large $n \in \mathbb{N}$, it follows that

$$
E\left(t ;-S_{0}\right) \Gamma= \begin{cases}\lim _{n \rightarrow \infty}\left(I+\frac{t}{n} S_{0}\right)^{-n} E\left(0^{+} ;-S_{0}\right) \Gamma, & t>0, \\ \lim _{n \rightarrow \infty}\left(I+\frac{t}{n} S_{0}\right)^{-n} E\left(0^{-} ;-S_{0}\right) \Gamma, & t<0,\end{cases}
$$

in the operator norm. Since $\left(I+\frac{t}{n} S_{0}\right)^{-n} E\left(0^{ \pm} ;-S_{0}\right) \Gamma$ is compact for $( \pm t)>0$, it follows that $E\left(t ;-S_{0}\right) \Gamma$ is compact for $0 \neq t \in \mathbb{R}$, which completes the proof. 


\subsection{Canonical factorization and matching of subspaces}

Let $-S_{0}$ be exponentially dichotomous and $\Gamma$ a bounded operator on a complex Banach space $\mathcal{X}$, and let $-S=-S_{0}+\Gamma$, where $\mathcal{D}(S)=\mathcal{D}\left(S_{0}\right)$ and $\{\lambda \in \mathbb{C}$ : $|\operatorname{Re} \lambda| \leq \varepsilon\} \subset \rho(S)$ for some $\varepsilon>0$. Then $-S$ is exponentially dichotomous if $E\left(t ;-\bar{S}_{0}\right) \Gamma$ is continuous in $0 \neq t \in \mathbb{R}$ in the operator norm. In this section we consider the analogous vector-valued Wiener-Hopf integral equation

$$
\phi(t)-\int_{0}^{\infty} E\left(t-\tau ;-S_{0}\right) \Gamma \phi(\tau) d \tau=g(t)
$$

where $t>0$.

Suppose $W$ is a continuous function from the extended imaginary axis $i(\mathbb{R} \cup$ $\{\infty\}$ ) into $\mathcal{L}(\mathcal{X})$. Then by a left canonical (Wiener-Hopf) factorization of $W$ we mean a representation of $W$ of the form

$$
W(\lambda)=W_{+}(\lambda) W_{-}(\lambda), \quad \operatorname{Re} \lambda=0,
$$

in which $W_{ \pm}( \pm \lambda)$ is continuous on the closed right half-plane (the point at $\infty$ included), is analytic on the open right half-plane, and takes only invertible values for $\lambda$ in the closed right half-plane (the point at infinity included). Obviously, such an operator function only takes invertible values on the extended imaginary axis. By a right canonical (Wiener-Hopf) factorization we mean a representation of $W$ of the form

$$
W(\lambda)=W_{-}(\lambda) W_{+}(\lambda), \quad \operatorname{Re} \lambda=0,
$$

where $W_{ \pm}(\lambda)$ are as above.

Theorems 6 and 7 and Corollary 8 of [14] can now easily be generalized with exactly the same proofs. We now require $-S_{0}$ to be an exponentially dichotomous and $\Gamma$ a bounded operator on a complex Banach space $\mathcal{X}$ (Hilbert space when generalizing Corollary 8 of [14]) such that $E\left(t ;-S_{0}\right) \Gamma$ is continuous in $0 \neq t \in \mathbb{R}$ in the operator norm, instead of requiring that either (i) $E\left(t ;-S_{0}\right)$ itself is continuous in the operator norm for $0 \neq t \in \mathbb{R}$ or (ii) $\Gamma$ is a compact operator. Lemma 2.1 then enables us to apply Theorems 6 and 7 and Corollary 8 of [14] in the case $\left(\lambda-S_{0}\right)^{-1} \Gamma$ is a compact operator for imaginary $\lambda$.

The above generalizations of Theorems 6 and 7 of [14] yield results on the equivalence of (i) left (resp., right) canonical Wiener-Hopf factorizability of $W(\lambda)=$ $\left(\lambda-S_{0}\right)^{-1}(\lambda-S)$, (ii) the complementarity in $\mathcal{X}$ of the range of one of the separating projections $P_{0}$ and $P$ and the kernel of the other, and (iii) the unique solvability of the vector-valued convolution equation on the positive (resp. negative) half-line with convolution kernel $E\left(\cdot ;-S_{0}\right) \Gamma$. The above generalization of Corollary 8 of [14] yields left and right canonical factorizability if the symbol $W(\lambda)=\left(\lambda-S_{0}\right)^{-1}(\lambda-S)$ of the half-line convolution equation involved is either close to the identity operator or has a strictly positive definite real part. Similar results in various different contexts exist in the finite-dimensional case [1], for equations with symbols analytic in a strip and at infinity [3], for extended Pritchard-Salamon realizations [8], and for abstract kinetic equations [7]. 


\subsection{Block operators}

Suppose $-S_{0}$ is exponentially dichotomous and $\Gamma$ is a bounded linear operator on a complex Banach space $\mathcal{X}$. Define $S$ by $-S=-S_{0}+\Gamma$, and put

$$
\mathcal{X}^{ \pm}=\operatorname{Im} E\left(0^{ \pm} ;-S_{0}\right),
$$

i.e., $\mathcal{X}^{+}=\operatorname{Im}\left(I-P_{0}\right)=\operatorname{Ker} P_{0}$ and $X^{-}=\operatorname{Im} P_{0}$. Assuming that $\Gamma\left[\mathcal{X}^{ \pm}\right] \subset \mathcal{X}^{\mp}$, we have the following block decompositions of $S_{0}$ and $S$ with respect to the direct $\operatorname{sum} \mathcal{X}=\mathcal{X}^{+} \dot{+} \mathcal{X}^{-}$:

$$
S_{0}=\left(\begin{array}{cc}
A_{0} & 0 \\
0 & -A_{1}
\end{array}\right), \quad S=\left(\begin{array}{cc}
A_{0} & -D \\
-Q & -A_{1}
\end{array}\right),
$$

where $-A_{0}$ and $-A_{1}$ are the generators of uniformly exponentially stable $C_{0}$ semigroups and $Q: \mathcal{X}^{+} \rightarrow \mathcal{X}^{-}$and $D: \mathcal{X}^{-} \rightarrow \mathcal{X}^{+}$are bounded. Then we call $S$ written in the form (2.9) a block operator, which is in line with the definition used in [14]. In the literature the notion of a block operator is also used in a wider sense (e.g., without assumptions about semigroup generators).

Theorem 9 of [14] can now be generalized in the same way without changing its proof. We now require $-S_{0}$ to be an exponentially dichotomous operator and $\Gamma$ a bounded operator on a complex Banach space $\mathcal{X}$ satisfying $\Gamma\left[\mathcal{X}^{ \pm}\right] \subset \mathcal{X}^{\mp}$, instead of requiring that either (i) $E\left(t ;-S_{0}\right)$ itself is continuous in the operator norm for $0 \neq t \in \mathbb{R}$ or (ii) $\Gamma$ is a compact operator. Here $\mathcal{X}^{+}$and $\mathcal{X}^{-}$are the kernel and range of the separating projection of $-S_{0}$, respectively.

The above generalization of Theorem 9 of [14] states that there exists a bounded linear operator $\Pi_{+}$from $\mathcal{X}^{-}$into $\mathcal{X}^{+}$which maps $\mathcal{D}\left(A_{1}\right)$ into $\mathcal{D}\left(A_{0}\right)$, has the property that $B_{1}=A_{1}+Q \Pi_{+}$generates an exponentially stable semigroup on $\mathcal{X}^{-}$, and satisfies the Riccati equation

$$
A_{0} \Pi_{+} x+\Pi_{+} A_{1} x-D x+\Pi_{+} Q \Pi_{+} x=0, \quad x \in \mathcal{D}\left(A_{1}\right),
$$

if and only if the equivalent statements (a)-(e) of Theorem 7 of [14] are true. Analogously, it states that there exists a bounded linear operator $\Pi_{-}$from $\mathcal{X}^{+}$into $\mathcal{X}^{-}$ which maps $\mathcal{D}\left(A_{0}\right)$ into $\mathcal{D}\left(A_{1}\right)$, has the property that $B_{0}=A_{0}-D \Pi_{\text {- generates }}$ an exponentially stable semigroup on $\mathcal{X}^{+}$, and satisfies the Riccati equation

$$
\Pi_{-} A_{0} x+A_{1} \Pi_{-} x-\Pi_{-} D \Pi_{-} x+Q x=0, \quad x \in \mathcal{D}\left(A_{0}\right) .
$$

if and only if the equivalent statements (a)-(e) of Theorem 8 of [14] are true. Similar results are valid in the finite-dimensional case [1] and for extended PritchardSalamon realizations $[8]$.

\section{Analytic bisemigroups and unbounded perturbations}

\subsection{Preliminaries on analytic semigroups}

As in [5] (but in contrast to the definition given in [12]), a closed linear operator $A$ densely defined on a complex Banach space $\mathcal{X}$ is called sectorial if there exists 
a $\delta$ with $0<\delta \leq(\pi / 2)$ such that the sector

$$
\Sigma_{\frac{\pi}{2}+\delta}=\left\{\lambda \in \mathbb{C}:|\arg \lambda|<\frac{\pi}{2}+\delta\right\} \backslash\{0\}
$$

is contained in the resolvent set of $A$, and if for each $\zeta \in(0, \delta)$ there exists $M_{\zeta} \geq 1$ such that

$$
\left\|(\lambda-A)^{-1}\right\| \leq \frac{M_{\zeta}}{|\lambda|}, \quad \lambda \in \overline{\Sigma_{\frac{\pi}{2}+\delta-\zeta}} \backslash\{0\} .
$$

According to [5], Theorem II 4.6, the sectorial operators are exactly the generators of bounded analytic semigroups. Thus $A$ is the generator of a uniformly exponentially stable analytic semigroup if and only if there exist $\delta$ and $\gamma$ with $0<\delta \leq(\pi / 2)$ and $\gamma>0$ such that (1) the sector

$$
-\gamma+\Sigma_{\frac{\pi}{2}+\delta}=\left\{\lambda \in \mathbb{C}:|\arg (\lambda+\gamma)|<\frac{\pi}{2}+\delta\right\} \backslash\{-\gamma\}
$$

is contained in the resolvent set of $A$, and (2) for each $\zeta \in(0, \delta)$ there exists $M_{\zeta} \geq 1$ such that

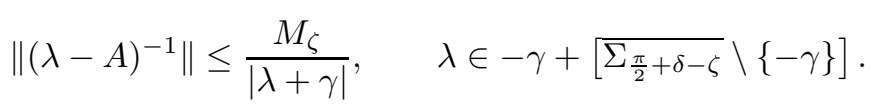

\subsection{Perturbation results for analytic bisemigroups}

A bisemigroup is called analytic if its constituent semigroups are analytic. Writing $-S$ for its generator and $P$ for its separating projection, we can define

$$
H(t ;-S)=\left\{\begin{array}{cc}
S e^{-t S}(I-P), & t>0 \\
-S e^{-t S} P, & t<0
\end{array}\right.
$$

for the derivative of $E(t ;-S)$ with respect to $0 \neq t \in \mathbb{R}$.

Next, we note that the generator $-S$ has the following two properties (cf. $(3.1)-(3.2))$ :

1. there exist $\delta$ and $\gamma$ with $0<\delta \leq(\pi / 2)$ and $\gamma>0$ such that the set

$$
\Omega_{\delta, \gamma}=\left\{\lambda \in \mathbb{C}:\left|\frac{\pi}{2}-\arg \lambda\right|<\delta \text { or }|\operatorname{Re} \lambda|<\gamma\right\}
$$

is contained in the resolvent set of $S$, and

2. for each $\zeta \in(0, \delta)$ there exists $N_{\zeta} \geq 1$ such that

$$
\left\|(\lambda-S)^{-1}\right\| \leq N_{\zeta}\left(\frac{1}{|\lambda+\gamma|}+\frac{1}{|\lambda-\gamma|}\right), \quad \lambda \in \overline{\Omega_{\zeta, \gamma}} \backslash\{\gamma,-\gamma\} .
$$

It is not clear if a closed and densely defined linear operator $-S$ on $\mathcal{X}$ having the properties (3.3)-(3.4) generates an analytic bisemigroup.

Starting from an exponentially dichotomous operator $-S_{0}$ on a complex Banach space $\mathcal{X}$ generating an analytic bisemigroup and a bounded linear operator $\Delta$ on $\mathcal{X}$, we now study sufficient conditions under which the unbounded perturbation $-S=-S_{0}+\Gamma$ of $-S_{0}$ for which $\Gamma=S_{0} \Delta$, is a generator of an analytic bisemigroup. We will always assume that $1 \notin \sigma(\Delta)$ and define $-S$ by

$$
\mathcal{D}(S)=(I-\Delta)^{-1}\left[\mathcal{D}\left(S_{0}\right)\right], \quad-S=-S_{0}(I-\Delta)
$$


Before deriving our main perturbation result, we prove the following lemma.

Lemma 3.1. Let $-S_{0}$ be the generator of an analytic bisemigroup and $\Delta$ a bounded linear operator such that $1 \notin \sigma(\Delta)$. Suppose that

1. there exist $\delta$ and $\gamma$ with $0<\delta \leq(\pi / 2)$ and $\gamma>0$ such that the set $\Omega_{\delta, \gamma}$ defined by (3.3) is contained in the resolvent set of $S=S_{0}(I-\Delta)$, and

2. $\int_{-\infty}^{\infty}\left\|H\left(t ;-S_{0}\right) \Delta\right\| d t<\infty$.

Then $-S$ is the generator of an analytic bisemigroup.

Proof. There exists $\varepsilon>0$ such that (2.3) is true. Using the resolvent identity (2.4), we obtain the convolution integral equation

$$
E(t ;-S) x-\int_{-\infty}^{\infty} H\left(t-\tau ;-S_{0}\right) \Delta E(\tau ;-S) x d \tau=E\left(t ;-S_{0}\right) x,
$$

where $x \in \mathcal{H}$ and $0 \neq t \in \mathbb{R}$. By assumption, in (3.5) the convolution kernel $H\left(\cdot ;-S_{0}\right) \Delta$ is continuous in the norm except for a jump discontinuity in $t=0$ and satisfies $\int_{-\infty}^{\infty} e^{\varepsilon|t|}\left\|H\left(t ;-S_{0}\right) \Delta\right\| d t<\infty$. Indeed, the integral is an improper integral at 0 and at $\pm \infty$. Convergence at $t=0$ is guaranteed by the second assumption. Convergence at $\pm \infty$ follows from (2.3) and a line of argument as on page 103 (bottom) of [5], which together prove that $H\left(t ;-S_{0}\right)$ is exponentially decaying. Thus $e^{\varepsilon|\cdot|} H\left(\cdot ;-S_{0}\right) \Delta$ is Bochner integrable.

The symbol of the convolution integral equation (3.5), which equals $I-\Delta+$ $\lambda\left(\lambda-S_{0}\right)^{-1} \Delta=\left(\lambda-S_{0}\right)^{-1}(\lambda-S)$, tends to $I$ in the norm as $\lambda \rightarrow \infty$ in the strip $|\operatorname{Re} \lambda|<\varepsilon$, because of the Riemann-Lebesgue lemma. Thus there exists $\varepsilon_{0} \in$ $(0, \min (\varepsilon, \gamma)]$ such that the symbol only takes invertible values on the strip $|\operatorname{Re} \lambda| \leq$ $\varepsilon_{0}$. By the Bochner-Phillips theorem [4], the convolution equation (3.5) has a unique solution $u(\cdot ; x)=E(\cdot ;-S) x$ with the following properties:

1) $E(\cdot ;-S)$ is strongly continuous, except for a jump discontinuity at $t=0$,

2) $\int_{-\infty}^{\infty} e^{\varepsilon_{0}|t|}\|E(t ;-S)\| d t<\infty$; hence $E(\cdot ;-S)$ is exponentially decaying,

3) the identity (2.2) holds.

As a result [2], $-S$ is exponentially dichotomous.

The following result has been established in [7] for the case in which $S_{0}$ is the inverse of a bounded and injective selfadjoint operator on a Hilbert space. In [7] it has been sketched how the arguments used to prove the Hilbert space case can also be applied to prove the Banach space case, without rendering details.

Theorem 3.2. Let $-S_{0}$ be the generator of an analytic bisemigroup and $\Delta$ a compact operator such that $1 \notin \sigma(\Delta)$ and $S=S_{0}(I-\Delta)$ does not have purely imaginary eigenvalues. Suppose that

$$
\int_{-\infty}^{\infty}\left\|H\left(t ;-S_{0}\right) \Delta\right\| d t<\infty
$$

Then $-S$ is the generator of an analytic bisemigroup. 
Proof. It suffices to prove the first condition of Lemma 3.1. Indeed, since the symbol of the convolution equation (3.5) is a compact perturbation of the identity and is invertible on a strip $|\operatorname{Re} \lambda| \leq \varepsilon_{0}$ about the imaginary axis while it has invertible limits in the operator norm as $\lambda \rightarrow 0$ and $\lambda \rightarrow \pm i \infty$, the spectrum of $S$ in this strip must consist of finitely many normal eigenvalues. Thus the first condition of Lemma 3.1 amounts to requiring the absence of purely imaginary eigenvalues of $S$, as assumed.

It is clear from the proof that the hypotheses of Theorem 3.2 can be replaced by the hypotheses that $-S_{0}$ is the generator of an analytic bisemigroup, $(\lambda-$ $\left.S_{0}\right)^{-1} \Delta$ is compact for purely imaginary $\lambda, 1 \notin \sigma(\Delta), S=S_{0}(I-\Delta)$ does not have purely imaginary eigenvalues, and (3.6) holds. It is not necessary to have $\Delta$ itself compact.

It is well known that sectorial operators have fractional powers [12]. Thus generators $-S=\left(-A_{0}\right) \dot{+} A_{1}$ of analytic bisemigroups, where $-A_{0}$ and $-A_{1}$ are generators of uniformly exponentially stable analytic semigroups, have fractional powers defined by $|S|^{\alpha} \stackrel{\text { def }}{=}\left(-A_{0}\right)^{\alpha} \dot{+}\left(-A_{1}\right)^{\alpha}$ for any $\alpha \in \mathbb{R}$. Moreover,

$$
\begin{aligned}
\left\||S|^{\alpha} E(t ;-S)\right\| & =O\left(|t|^{-\alpha}\right), & & t \rightarrow 0^{ \pm} ; \\
\exists c>0:\left\||S|^{\alpha} E(t ;-S)\right\| & =O\left(|t|^{-\alpha} e^{-c|t|}\right), & & t \rightarrow \pm \infty .
\end{aligned}
$$

As a result of (3.7)-(3.8) we have

$$
\begin{aligned}
\left\||S|^{-\alpha} H(t ;-S)\right\| & =O\left(|t|^{\alpha-1}\right), & & t \rightarrow 0^{ \pm} \\
\exists c>0:\left\||S|^{-\alpha} H(t ;-S)\right\| & =O\left(|t|^{\alpha-1} e^{-c|t|}\right), & & t \rightarrow \pm \infty .
\end{aligned}
$$

The following corollary is now clear.

Corollary 3.3. Let $-S_{0}$ be the generator of an analytic bisemigroup and $\Delta$ a compact operator such that $1 \notin \sigma(\Delta), S=S_{0}(I-\Delta)$ does not have purely imaginary eigenvalues, and $\operatorname{Im} \Delta \subset \mathcal{D}\left(\left|S_{0}\right|^{\alpha}\right)$ for some $\alpha>0$. Then $-S$ is the generator of an analytic bisemigroup.

\subsection{Canonical factorization and matching of subspaces}

The following results can all be found in [7] for the case in which $S_{0}$ is the inverse of a bounded and injective selfadjoint operator on a Hilbert space.

Theorem 3.4. Suppose $\mathcal{X}$ is a complex Banach space. Let $-S_{0}$ be the generator of an analytic bisemigroup and $\Delta$ a bounded operator with $1 \notin \sigma(\Delta)$ such that $\left(\lambda-S_{0}\right)^{-1} \Delta$ is compact for purely imaginary $\lambda, S_{0}(I-\Delta)$ does not have purely imaginary eigenvalues, and (3.6) is true. Let $P_{0}$ and $P$ stand for the separating projections of $-S_{0}$ and $-S$, respectively. Then the following statements are equivalent:

(a) The operator function

$$
\begin{aligned}
& W(\lambda)=\left(\lambda-S_{0}\right)^{-1}(\lambda-S)=I_{\mathcal{X}}-\Delta+\lambda\left(\lambda-S_{0}\right)^{-1} \Delta, \quad|\operatorname{Re} \lambda| \leq \varepsilon, \\
& \text { has a left canonical factorization with respect to the imaginary axis. }
\end{aligned}
$$


(b) We have the decomposition

$$
\operatorname{Ker} P \dot{+} \operatorname{Im} P_{0}=\mathcal{X} .
$$

(c) For some (and hence every) $E\left(\mathbb{R}^{+} ; \mathcal{X}\right)$, the vector-valued Wiener-Hopf equation

$$
\phi(t)-\int_{0}^{\infty} H\left(t-\tau ;-S_{0}\right) \Delta \phi(\tau) d \tau=g(t), \quad t>0,
$$

is uniquely solvable in $E\left(\mathbb{R}^{+} ; \mathcal{X}\right)$ for any $g \in E\left(\mathbb{R}^{+} ; \mathcal{X}\right)$.

Theorem 3.5. Suppose $\mathcal{X}$ is a complex Banach space. Let $-S_{0}$ be the generator of an analytic bisemigroup and $\Delta$ a bounded operator with $1 \notin \sigma(\Delta)$ such that $\left(\lambda-S_{0}\right)^{-1} \Delta$ is compact for purely imaginary $\lambda, S_{0}(I-\Delta)$ does not have purely imaginary eigenvalues, and (3.6) is true. Let $P_{0}$ and $P$ stand for the separating projections of $-S_{0}$ and $-S$, respectively. Then the following statements are equivalent:

(a) The operator function

$$
W(\lambda)=\left(\lambda-S_{0}\right)^{-1}(\lambda-S)=I_{\mathcal{X}}-\Delta+\lambda\left(\lambda-S_{0}\right)^{-1} \Delta, \quad|\operatorname{Re} \lambda| \leq \varepsilon,
$$

has a right canonical factorization with respect to the imaginary axis.

(b) We have the decomposition

$$
\operatorname{Ker} P_{0} \dot{+} \operatorname{Im} P=\mathcal{X} .
$$

(c) For some (and hence every) $E\left(\mathbb{R}^{-} ; \mathcal{X}\right)$, the vector-valued Wiener-Hopf equation

$$
\phi(t)-\int_{-\infty}^{0} H\left(t-\tau ;-S_{0}\right) \Delta \phi(\tau) d \tau=g(t), \quad t<0,
$$

is uniquely solvable in $E\left(\mathbb{R}^{-} ; \mathcal{X}\right)$ for any $g \in E\left(\mathbb{R}^{-} ; \mathcal{X}\right)$.

Corollary 3.6. Suppose $\mathcal{H}$ is a complex Hilbert space. Let $-S_{0}$ be the generator of an analytic bisemigroup and $\Delta$ a bounded operator with $1 \notin \sigma(\Delta)$ such that $\left(\lambda-S_{0}\right)^{-1} \Delta$ is compact for purely imaginary $\lambda, S_{0}(I-\Delta)$ does not have purely imaginary eigenvalues, and (3.6) is true. Let $P_{0}$ and $P$ be the separating projections of $-S_{0}$ and $-S$, respectively. Suppose

$$
\sup _{\operatorname{Re} \lambda=0}\left\|-\Delta+\lambda\left(\lambda-S_{0}\right)^{-1} \Delta\right\|<1
$$

Then all of the following statements are true:

(a) The operator function $W(\cdot)$ in (3.9) has a left and a right canonical factorization with respect to the imaginary axis.

(b) We have the decompositions (3.10) and (3.12).

(c) For some (and hence every) $E\left(\mathbb{R}^{ \pm} ; \mathcal{H}\right)$, the vector-valued Wiener-Hopf equation (3.11) [(3.13), respectively] is uniquely solvable in $E\left(\mathbb{R}^{ \pm} ; \mathcal{H}\right)$ for any $g \in E\left(\mathbb{R}^{ \pm} ; \mathcal{H}\right)$. 


\section{Acknowledgment}

The authors are greatly indebted to Prof. Karl-Heinz Förster for a question suggesting a generalization of previous results on bisemigroup perturbation.

\section{References}

[1] H. Bart, I. Gohberg, and M.A. Kaashoek, Minimal Factorization of Matrix and Operator Functions. Birkhäuser OT 1, Basel and Boston, 1979.

[2] H. Bart, I. Gohberg, and M.A. Kaashoek, Wiener-Hopf factorization, inverse Fourier transforms and exponentially dichotomous operators. J. Funct. Anal. 68 (1986), 1-42.

[3] H. Bart, I. Gohberg, and M.A. Kaashoek, Wiener-Hopf equations with symbols analytic in a strip. In: I. Gohberg and M.A. Kaashoek, eds., Constructive Methods of Wiener-Hopf Factorization. Birkhäuser OT 21, Basel, 1986, pp. 39-74.

[4] S. Bochner and R.S. Phillips, Absolutely convergent Fourier expansions for non-commutative normed rings. Ann. Math. 43 (1942), 409-418.

[5] K.-J. Engel and R. Nagel, One-parameter Semigroups for Linear Evolution Equations. Springer GTM 194, Berlin, 2000.

[6] I.C. Gohberg and J. Leiterer, Factorization of operator functions with respect to a contour. II. Canonical factorization of operator functions close to the identity. Math. Nachrichten 54 (1972), 41-74 [Russian].

[7] W. Greenberg, C.V.M. van der Mee, and V. Protopopescu, Boundary Value Problems in Abstract Kinetic Theory. Birkhäuser OT 23, Basel and Boston, 1987.

[8] M.A. Kaashoek, C.V.M. van der Mee, and A.C.M. Ran, Wiener-Hopf factorization of transfer functions of extended Pritchard-Salamon realizations. Math. Nachrichten 196 (1998), 71-102.

[9] H. Langer, A.C.M. Ran, and B.A. van de Rotten, Invariant subspaces of infinitedimensional Hamiltonians and solutions of the corresponding Riccati equations. In: I. Gohberg and H. Langer, eds., Linear Operators and Matrices. Birkhäuser OT 130, Basel and Boston, 2001, pp. 235-254.

[10] H. Langer and C. Tretter, Spectral decomposition of some nonselfadjoint block operator matrices. J. Operator Theory 39 (1998), 339-359.

[11] H. Langer and C. Tretter, Diagonalization of certain block operator matrices and applications to Dirac operators. In: H. Bart, I. Gohberg, and A.C.M. Ran, eds., Operator Theory and Analysis. Birkhäuser OT 122, Basel and Boston, 2001, pp. 331-358.

[12] A. Lunardi, Analytic Semigroups and Optimal Regularity in Parabolic Problems, Birkhäuser PNDEA 16, Basel and Boston, 1995.

[13] C.V.M. van der Mee, Transport theory in $L_{p}$-spaces. Integral Equations and Operator Theory 6 (1983), 405-443.

[14] A.C.M. Ran and C. van der Mee, Perturbation results for exponentially dichotomous operators on general Banach spaces. J. Func. Anal. 210 (2004), 193-213. 
Cornelis V.M van der Mee

Dipartimento di Matematica e Informatica

Università di Cagliari

Viale Merello 92

I-09123 Cagliari, Italy

e-mail: cornelis@bugs . unica.it

André C.M. Ran

Afdeling Wiskunde, FEW

Vrije Universiteit

De Boelelaan 1081a

NL-1081 HV Amsterdam, The Netherlands

e-mail: ran@cs.vu.nl 